\title{
Production of the top-pions from the higgsless- top-Higgs model at the LHC
}

\author{
Chong-Xing Yue and Yi-Qun Di \\ Department of Physics, Liaoning Normal University, Dalian, 116029 P. R. China *
}

May 18, 2018

\begin{abstract}
The top-pions $\left(\pi_{t}^{0, \pm}\right)$ predicted by extra dimensional descriptions of the topcolor scenario have similar feature with those in four dimensional topcolor scenario, which have large Yukawa couplings to the third generation quarks. In the context of the higgsless-top-Higgs(HTH) model, we discuss the production of these new particles at the CERN Large Hadron Collider(LHC) via various suitable mechanisms (gluongluon fusion, bottom-bottom fusion, gluon-bottom fusion, and the usual Drell-Yan processes) and estimate their production rates. We find that, as long as the toppions are not too heavy, they can be abundantly produced at the LHC. The possible signatures of these new particles might be detected at the LHC experiments.
\end{abstract}

${ }^{*}$ E-mail:cxyue@lnnu.edu.cn 


\section{Introducton}

To completely avoid the problems arising from the elementary Higgs field in the standard model(SM), various models for dynamical electroweak symmetry breaking(EWSB) have been proposed, among which the topcolor scenario is attractive because it can explain the large top mass and provide a possible EWSB mechanism[1]. The common feature of this kind of models is that topcolor interactions make small contributions to EWSB and give rise to most part of the top mass, while EWSB is mainly induced by technicolor or other strong interactions.

Recently, the model building along with the TeV-scale extra dimensional scenario[2] has been widely studied. Considering the gauge symmetry breaking can be easily achieved by imposing appropriate boundary conditions[3], the higgsless theory[4] was proposed and the topcolor scenario was reconsidered in extra dimensions $[5,6]$. For extra dimensional descriptions of the topcolor scenario[5], there are two separate 5 dimensional anti de Sitter spaces $\left(A d S_{5}^{\prime} s\right)$ with each sector having its own corresponding infrared(IR) brane. The light fermions propagate in an $A d S_{5}$ sector, while the third generation quarks would propagate in other $A d S_{5}$ sector, and sources for EWSB are localized on both of these IR branes. Using the appropriate boundary and matching conditions, there are three possibilities for EWSB: Higgs-top-Higgs, higgsless-top-Higgs, and higgsless-higgsless. For the higgsless-top-Higgs case, most of EWSB comes from the higgsless sector, and the top quark gets its mass from a top-Higgs on the other IR brane.

Similarly to the topcolor scenario in four dimensional space, the higgsless-top-Higgs (HTH) model[5] predicts the existence of isotriplet scalars, called top-pions $\left(\pi_{t}^{0, \pm}\right)$, which have large Yukawa couplings to the third generation quarks and sensibly small couplings to the massive gauge bosons and light quarks. The top-pions get masses at one-loop from gauge interactions, which can propagate from one boundary to the other. The mass scale is set by the cutoff scale on the IR brane, so the top-pions should be quite heavy and can not be abundantly produced at the present high energy experiments. The aim of

this paper is to investigate production of these new particles $\left(\pi_{t}^{0, \pm}\right)$ at the CERN Large Hadron Collider(LHC) with $\sqrt{s}=14 T e V$ and see whether the possible signatures of the 
HTH model can be detected in the near future LHC experiments.

In the rest of this paper, we will give our results in detail. The couplings of the top-pions $\pi_{t}^{0, \pm}$ to the ordinary particles are given in Sec.II, and the decay widths of the possible decay modes are also estimated in this section. Sections III and IV are devoted to the computation of the production cross sections of $\pi_{t}^{0}$ and $\pi_{t}^{ \pm}$at the LHC, respectively. Some phenomenological analysis are also included in these sections. Our conclusions are given in Sec. V.

\section{The possible decay modes of the top-pions $\pi_{t}^{0, \pm}$}

For the HTH model[5], there are two $A d S_{5}$ spaces intersecting along a codimension one surface(Planck brane) that would serve as a ultraviolet(UV) cutoff of the two $A d S_{5}$ spaces. The two $A d S_{5}$ spaces, denoted as $A d S_{5}^{w}$ and $A d S_{5}^{t}$, are characterized by their own curvature scale $R_{w}$ and $R_{t}$, respectively. The common UV boundary for each brane is located at the point $Z=R_{w}$ or $R_{t}$ in the coordinate system and each $A d S_{5}$ space is also cut by an IR boundary located at $Z=R_{w}^{\prime}$ or $R_{t}^{\prime}$. The IR brane at $R_{w}^{\prime}$ has a higgsless boundary condition and is responsible for most of EWSB, while the top-Higgs $h_{t}^{0}$ on the IR brane at $R_{t}^{\prime}$ makes very small contributions to EWSB. Thus, for the HTH model, there is the following relation:

$$
\nu^{2}=\nu_{w}^{2}+\nu_{t}^{2}
$$

where $\nu=246 \mathrm{GeV}$ is the electroweak scale, $\nu_{w}$ represents the contributions of the higgsless sector to EWSB. The vacuum expectation value(VEV) $\nu_{t}$ of the top-Higgs produces all of the top mass. To produce the large top mass $m_{t}$, the VEV $\nu_{t}$ should be very small. Thus, the top-pions have large Yukawa couplings to the third generation quarks.

The coupling constants of the top-pions $\pi_{t}^{0, \pm}$ to the third generation quarks can be written as[5]:

$$
\begin{gathered}
\pi_{t}^{0} t \bar{t}: \frac{m_{t}}{\nu_{t}}, \quad \pi_{t}^{0} b \bar{b}: \frac{m_{b}}{\nu_{t}} \\
\pi_{t}^{-} t \bar{b}: \frac{e}{\sqrt{2} S_{W} m_{W}} \frac{\nu}{\nu_{t}}\left[m_{t} P_{R}+m_{b} P_{L}\right]
\end{gathered}
$$

where $S_{W}=\sin \theta_{W}, \theta_{W}$ is the Weinberg angle, and $P_{L(R)}=\left(1 \mp \gamma_{5}\right) / 2$ is the left(right)handed projection operator. Certainly, there might be the flavor changing(FC) couplings 
$\pi_{t}^{0} \bar{t} c$ and $\pi_{t}^{-} \bar{b} c$. However, for the HTH model, the flavor physics is generated on the Planck brane via mixing in non-diagonal localized kinetic terms, one can choose the free parameters to avoid the presence of the FC couplings $\pi_{t}^{0} \bar{t} c$ and $\pi_{t}^{-} \bar{b} c$. Furthermore, considering the electorweak precision measurement constraints, the top-pions should be quite heavy. Through out this paper, we will take the top-pion mass in the range of $400 \mathrm{GeV} \sim 800 \mathrm{GeV}$. In this case, the main decay channels are $\pi_{t}^{0} \rightarrow t \bar{t}$ and $\pi_{t}^{ \pm} \rightarrow t b$. Thus, we will not consider the FC couplings of $\pi_{t}^{0, \pm}$ in this paper. Compared to the SM Higgs boson, the couplings of $\pi_{t}^{0}$ to the third generation quarks are enhanced by the factor $\nu / \nu_{t}$, while the tree-level couplings of $\pi_{t}^{0}$ to the electroweak gauge bosons $W$ and $Z$ are suppressed by the factor $\nu_{t} / \nu$ :

$$
\pi_{t}^{0} Z Z: \frac{\nu_{t}}{\nu} \frac{e m_{Z}}{S_{W} C_{W}} g_{\mu \nu}, \quad \pi_{t}^{0} W^{+} W^{-}: \frac{\nu_{t}}{\nu} \frac{e m_{W}}{S_{W}} g_{\mu \nu}
$$

For the neutral top-pion $\pi_{t}^{0}$ decays, we will focus our attention on the decay modes: $t \bar{t}, b \bar{b}, W^{+} W^{-}, Z Z, g g$ and $\gamma \gamma$. The expressions of the decay widths for these decay channels can be written as:

$$
\begin{aligned}
& \Gamma\left(\pi_{t}^{0} \rightarrow t \bar{t}\right)=\frac{3 m_{t}^{2}}{8 \pi \nu_{t}^{2}} m_{\pi_{t}}\left(1-\frac{4 m_{t}^{2}}{m_{\pi_{t}}^{2}}\right)^{\frac{3}{2}} \\
& \Gamma\left(\pi_{t}^{0} \rightarrow b \bar{b}\right)=\frac{3 m_{b}^{2}}{8 \pi \nu_{t}^{2}} m_{\pi_{t}}\left(1-\frac{4 m_{b}^{2}}{m_{\pi_{t}}^{2}}\right)^{\frac{3}{2}} \\
& \Gamma\left(\pi_{t}^{0} \rightarrow W^{+} W^{-}\right)=\frac{\alpha_{e} m_{\pi_{t}}^{3}}{16 S_{W}^{2} m_{W}^{2}}\left(\frac{\nu_{t}}{\nu}\right)^{2}\left(1-\frac{4 m_{W}^{2}}{m_{\pi_{t}}^{2}}\right)^{\frac{1}{2}}\left(1-4 \frac{m_{W}^{2}}{m_{\pi_{t}}^{2}}+12 \frac{m_{W}^{4}}{m_{\pi_{t}}^{4}}\right), \\
& \Gamma\left(\pi_{t}^{0} \rightarrow Z Z\right)=\frac{\alpha_{e} m_{\pi_{t}}^{3}}{32 S_{W}^{2} m_{W}^{2}}\left(\frac{\nu_{t}}{\nu}\right)^{2}\left(1-\frac{4 m_{Z}^{2}}{m_{\pi_{t}}^{2}}\right)^{\frac{1}{2}}\left(1-4 \frac{m_{Z}^{2}}{m_{\pi_{t}}^{2}}+12 \frac{m_{Z}^{4}}{m_{\pi_{t}}^{4}}\right), \\
& \Gamma\left(\pi_{t}^{0} \rightarrow g g\right)=\frac{2 \alpha_{s}^{2} m_{\pi_{t}}^{3} m_{t}^{4}}{9 \nu_{t}^{2} \pi^{3}}\left[\left(1-4 \frac{m_{t}^{2}}{m_{\pi_{t}}^{2}}\right) C_{0}\left(m_{t}^{2}\right)-\frac{2}{m_{\pi_{t}}^{2}}\right]^{2}, \\
& \Gamma\left(\pi_{t}^{0} \rightarrow \gamma \gamma\right)=\frac{\alpha_{e}^{2} m_{\pi_{t}}^{3}}{16 \pi^{3}}\left\{\frac{4}{3 \nu_{t}^{2}}\left[m_{t}^{2}\left(1-4 \frac{m_{t}^{2}}{m_{\pi_{t}}^{2}}\right) C_{0}\left(m_{t}^{2}\right)-\frac{2 m_{t}^{2}}{m_{\pi_{t}}^{2}}\right]\right. \\
& \left.+\frac{\nu_{t}^{2}}{\nu^{4}}\left[\frac{1}{2}+3 \frac{m_{W}^{2}}{m_{\pi_{t}}^{2}}-3 m_{W}^{2}\left(1-2 \frac{m_{W}^{2}}{m_{\pi_{t}}^{2}}\right) C_{0}\left(m_{W}^{2}\right)\right]\right\}^{2} .
\end{aligned}
$$


The expression of the three-point scalar integral $C_{0}\left(m_{i}^{2}\right)$ can be written as $C_{0}\left(m_{i}^{2}\right)=$ $C_{0}\left(m_{\pi_{t}}^{2}, 0,0, m_{i}^{2}, m_{i}^{2}, m_{i}^{2}\right)[7]$. For the decay channels $\pi_{t}^{0} \rightarrow g g$ and $\gamma \gamma$, we have neglected the contributions of the light quarks.

To obtain numerical results, we need to specify the relevant SM input parameters. We take the SM parameters as $m_{t}=172.7 \mathrm{GeV}[8], S_{W}^{2}=0.2315, \alpha_{e}=1 / 128.8, \alpha_{s}=$ $0.112, m_{Z}=91.187 \mathrm{GeV}$, and $m_{W}=80.425 \mathrm{GeV}[9]$. Except for these SM input parameters, the partial decay widths are dependent on the two free parameters $\nu_{t}$ and $m_{\pi_{t}}$. To make that the HTH model generates enough large top mass and satisfies the electroweak precision constraints, the top-Higgs VEV $\nu_{t}$ should be very small and the top-pion should be quite heavy[5]. As numerical estimation, we will take $\nu_{t} \leq 50 \mathrm{GeV}\left(\nu_{t} / \nu \leq 1 / 5\right)$ and assume that the top-pion mass $m_{\pi_{t}}$ is in the range of $400 \mathrm{GeV} \sim 800 \mathrm{GeV}$.

The branching ratios of the prominent decay channels for the neutral top-pion $\pi_{t}^{0}$ are pictured in Fig.1 as functions of the top-pion mass $m_{\pi_{t}}$ for $\nu_{t} / \nu \approx 1 / 5$, in which we have multiplied the factors 100 and 10 to the branching ratios $\operatorname{Br}\left(\pi_{t}^{0} \rightarrow b \bar{b}\right)\left[\operatorname{Br}\left(\pi_{t}^{0} \rightarrow\right.\right.$ $\left.\left.W^{+} W^{-}\right), \operatorname{Br}\left(\pi_{t}^{0} \rightarrow Z Z\right)\right]$ and $\operatorname{Br}\left(\pi_{t}^{0} \rightarrow g g\right)$, respectively. Since the value of the branching ratio $\operatorname{Br}\left(\pi_{t}^{0} \rightarrow \gamma \gamma\right)$ is largely smaller than that of $\operatorname{Br}\left(\pi_{t}^{0} \rightarrow t \bar{t}\right)$, so we have not shown $\operatorname{Br}\left(\pi_{t}^{0} \rightarrow \gamma \gamma\right)$ in Fig.1. From Fig.1 we can see that, although the decay widths for all of the possible decay channels increase as $m_{\pi_{t}}$ increasing, the main decay modes of $\pi_{t}^{0}$ are $t \bar{t}$ and $g g$. For $400 G e V \leq m_{\pi_{t}} \leq 800 G e V$, the value of $\operatorname{Br}\left(\pi_{t}^{0} \rightarrow g g\right)$ is in the range of $1.33 \% \sim 0.34 \%$. Certainly, the values of the branching ratios for various possible modes vary as the parameter $\nu_{t} / \nu$ varying. For example, the value of the branching ratio $\operatorname{Br}\left(\pi_{t}^{0} \rightarrow g g\right)$ increases from $1.33 \%$ to $1.35 \%$ as $\nu_{t} / \nu$ decreasing from $1 / 5$ to $1 / 8$. Thus the neutral top-pion $\pi_{t}^{0}$ might be abundantly produced at the LHC via the subprocess $g g \rightarrow \pi_{t}^{0}$. The values of the branching ratios for all possible decay modes are given in Table I for three values of the top-pion mass $m_{\pi_{t}}$ and the top-Higgs $V E V \nu_{t}$. 


\begin{tabular}{|c|c||c|c|c|c|c|}
\hline$\nu_{t}(\mathrm{GeV})$ & $m_{\pi_{t}}(\mathrm{GeV})$ & $\operatorname{Br}(t \bar{t})$ & $\operatorname{Br}(b \bar{b})$ & $\operatorname{Br}\left(W^{+} W^{-}\right)$ & $\operatorname{Br}(Z Z)$ & $\operatorname{Br}(g g)$ \\
\hline \multirow{3}{*}{50} & 400 & $96.69 \%$ & $0.56 \%$ & $0.97 \%$ & $0.45 \%$ & $1.33 \%$ \\
\cline { 2 - 7 } & 600 & $98.63 \%$ & $0.12 \%$ & $0.54 \%$ & $0.26 \%$ & $0.46 \%$ \\
\cline { 2 - 7 } & 800 & $98.47 \%$ & $0.09 \%$ & $0.74 \%$ & $0.36 \%$ & $0.34 \%$ \\
\hline \multirow{3}{*}{40} & 400 & $97.50 \%$ & $0.57 \%$ & $0.40 \%$ & $0.19 \%$ & $1.34 \%$ \\
\cline { 2 - 7 } & 600 & $90.09 \%$ & $0.12 \%$ & $0.22 \%$ & $0.11 \%$ & $0.46 \%$ \\
\cline { 2 - 7 } & 800 & $99.11 \%$ & $0.09 \%$ & $0.30 \%$ & $0.15 \%$ & $0.34 \%$ \\
\hline \multirow{3}{*}{30} & 400 & $97.89 \%$ & $0.57 \%$ & $0.13 \%$ & $0.06 \%$ & $1.35 \%$ \\
\cline { 2 - 7 } & 600 & $99.31 \%$ & $0.12 \%$ & $0.07 \%$ & $0.03 \%$ & $0.46 \%$ \\
\cline { 2 - 7 } & 800 & $99.42 \%$ & $0.09 \%$ & $0.10 \%$ & $0.05 \%$ & $0.34 \%$ \\
\hline
\end{tabular}

Table I: The values of leading branching ratios for three values of the top-Higgs $V E V$ $\nu_{t}$ and the mass parameter $m_{\pi_{t}}$.

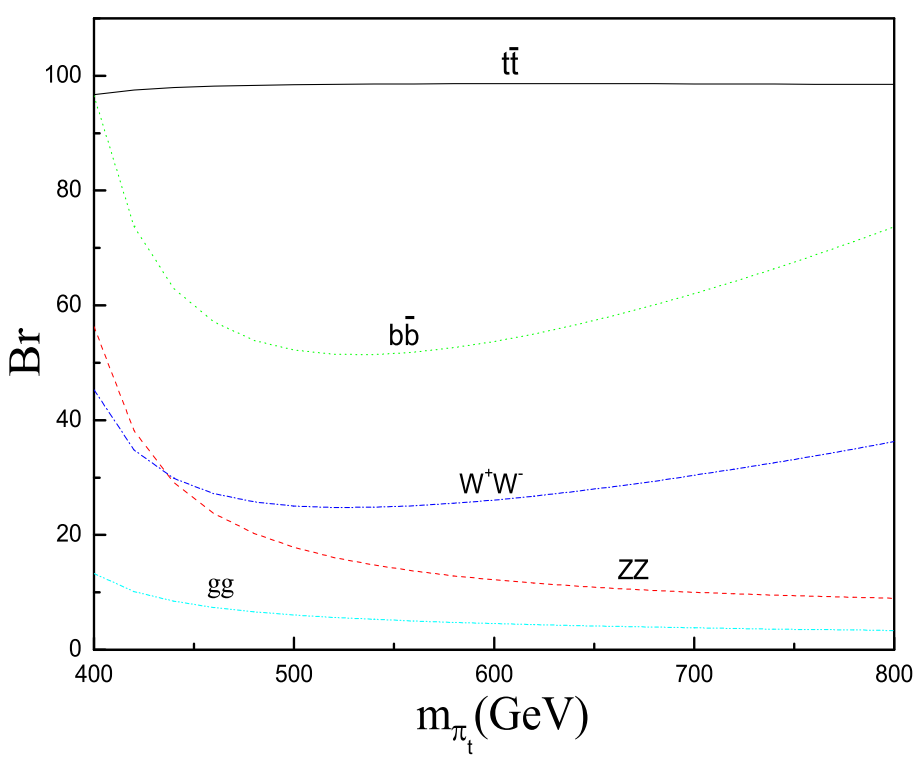

Figure 1: Branching ratios for the prominent decay modes of the neutral top-pion $\pi_{t}^{0}$ as functions of the mass parameter $m_{\pi_{t}}$ for $\nu_{t} / \nu \approx 1 / 5$.

For the charged top-pions $\pi_{t}^{ \pm}$, the mainly decay mode is $t b$. The decay width of the 
decay channel $\pi_{t}^{+} \rightarrow t \bar{b}$ can be written as:

$$
\begin{aligned}
\Gamma\left(\pi_{t}^{+} \rightarrow t \bar{b}\right)= & \frac{3 \alpha_{e}}{8 S_{W}^{2} m_{W}^{2} m_{\pi_{t}}^{3}}\left(\frac{\nu}{\nu_{t}}\right)^{2} \lambda^{\frac{1}{2}}\left(m_{\pi_{t}}^{2}, m_{t}^{2}, m_{b}^{2}\right) \\
& {\left[\left(m_{\pi_{t}}^{2}-m_{t}^{2}-m_{b}^{2}\right)\left(m_{t}^{2}+m_{b}^{2}\right)-4 m_{b}^{2} m_{t}^{2}\right], }
\end{aligned}
$$

where $\lambda(a, b, c)=(a-b-c)^{2}-4 b c$. Certainly, the charged top-pions $\pi_{t}^{ \pm}$can also decay to $W Z$ and $W \gamma$ at loop level. However, comparing with the tree-level process $\pi_{t}^{ \pm} \rightarrow t b$, the branching ratios of the one-loop processes $\pi_{t}^{ \pm} \rightarrow W Z, W \gamma$ are very small. The rare decay channels for the charged top-pions $\pi_{t}^{ \pm}$have been studied in the context of topcolor-assisted technicolor(TC2) models[10].

In the following sections, we will use above formulas to study the production of the top-pions $\pi_{t}^{0, \pm}$ at the LHC.

\section{Production of the neutral top-pion $\pi_{t}^{0}$ at the LHC}

From above discussions, we can see that the production of the neutral top-pion $\pi_{t}^{0}$ at the LHC mainly comes from the gluon fusion $\left(g g \rightarrow \pi_{t}^{0}\right)$ and the bottom quark fusion $\left(b b \rightarrow \pi_{t}^{0}\right)$. The production cross sections at parton level, which are proportional to the decay widths $\Gamma\left(\pi_{t}^{0} \rightarrow g g\right)$ and $\Gamma\left(\pi_{t}^{0} \rightarrow b \bar{b}\right)$, can be approximately written as:

$$
\begin{gathered}
\hat{\sigma}\left(g g \rightarrow \pi_{t}^{0}\right)=\frac{\alpha_{s}^{2} m_{t}^{4}}{64 \pi \nu_{t}^{2}}\left[\left(1-4 \frac{m_{t}^{2}}{m_{\pi_{t}}^{2}}\right) C_{0}\left(m_{t}^{2}\right)-\frac{2}{m_{\pi_{t}}^{2}}\right]^{2}, \\
\hat{\sigma}\left(b \bar{b} \rightarrow \pi_{t}^{0}\right)=\frac{3 \pi m_{b}^{2}}{4 \nu_{t}^{2} m_{\pi_{t}}} .
\end{gathered}
$$

The production cross section for the process $p p \rightarrow \pi_{t}^{0}+X$ at the LHC can be expressed as:

$$
\begin{aligned}
\sigma\left(p p \rightarrow \pi_{t}^{0}+X\right) & =\int_{0}^{1} d x_{1} \int_{0}^{1} d x_{2} f_{g / p}\left(x_{1}, \mu_{F}\right) f_{g / p}\left(x_{2}, m_{\pi_{t}}^{2}\right) \hat{\sigma}\left(g g \rightarrow \pi_{t}^{0}\right) \\
& +\int_{0}^{1} d x_{1} \int_{0}^{1} d x_{2} f_{b / p}\left(x_{1}, m_{\pi_{t}}^{2}\right) f_{b / p}\left(x_{2}, \mu_{F}\right) \hat{\sigma}\left(b \bar{b} \rightarrow \pi_{t}^{0}\right)
\end{aligned}
$$

where $f_{i / p}$ are the parton distribution functions(PDF's) for gluons and bottom quarks evaluated at some factorization scale $\mu_{F}$. In our numerical calculation, we will use CTEQ6L PDF's[11] for the quark and gluon PDF's. Following the suggestions given by Ref.[12], 
we assume that the factorization scale $\mu_{F}$ for the bottom quark PDF is of order $m_{\pi_{t}} / 4$ in this paper.

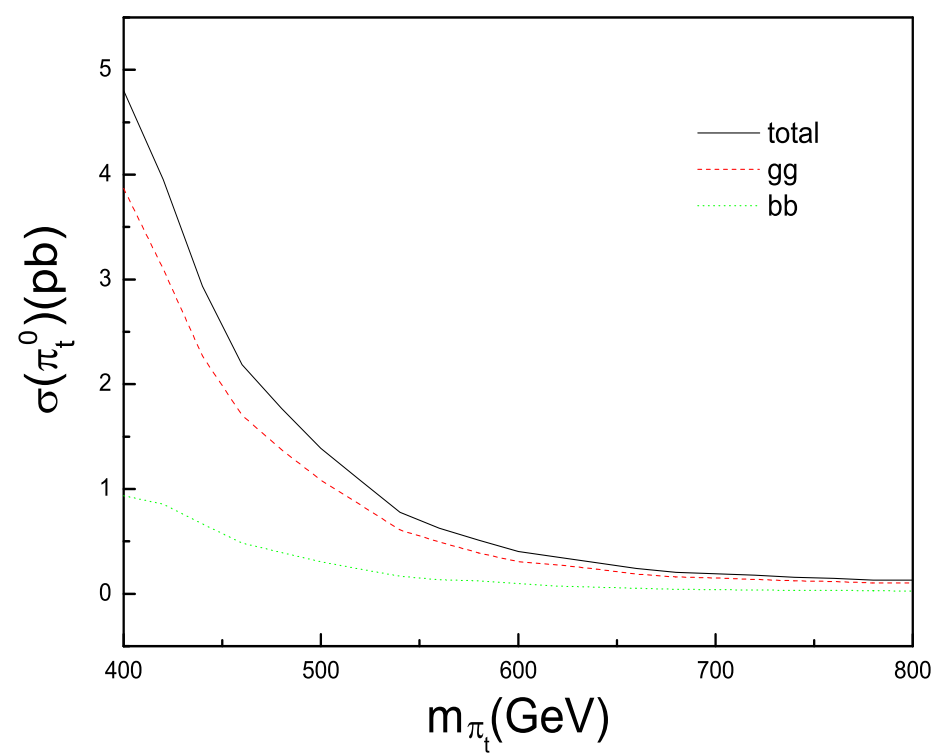

Figure 2: The production cross section $\sigma\left(\pi_{t}^{0}\right)$ at the LHC with $\sqrt{s}=14 T e V$ as a function of the mass parameter $m_{\pi_{t}}$ for $\nu_{t} / \nu \approx 1 / 5$.

The production cross section $\sigma\left(\pi_{t}^{0}\right)$ of the process $p p \rightarrow \pi_{t}^{0}+X$ at the LHC with $\sqrt{s}=14 T e V$ is plotted in Fig.2 as a function of the top-pion mass $m_{\pi_{t}}$ for $\nu_{t} / \nu \approx 1 / 5$, in which the dashed line, dotted line and solid line represent the contributions of the gluon fusion process, bottom quark fusion process, and both these processes, respectively. From Fig. 2 we can see that the production cross section $\sigma\left(\pi_{t}^{0}\right)$ mainly comes from the parton process $g g \rightarrow \pi_{t}^{0}$. However, the contributions of the process $b \bar{b} \rightarrow \pi_{t}^{0}$ to $\sigma\left(\pi_{t}^{0}\right)$ can not be neglected, which is in the range of $935 \mathrm{fb} \sim 27 \mathrm{fb}$ for $400 \mathrm{GeV} \leq m_{\pi_{t}} \leq 800 \mathrm{GeV}$. This is because the neutral top-pion $\pi_{t}^{0}$ has a enhanced coupling with bottom quark pair, $g_{\pi_{t}^{0} b \bar{b}}=\frac{m_{b}}{\nu_{t}}$. If we assume the yearly integrated luminosity $\mathcal{L}_{\text {int }}=100 \mathrm{fb}^{-1}$ for the LHC with $\sqrt{s}=14 \mathrm{TeV}$, then there will be $1.3 \times 10^{4} \sim 4.8 \times 10^{5} \pi_{t}^{0}$ events to be generated per year. Certainly, the numerical results increase as the $V E V \nu_{t}$ decreasing. For example, if we assume $\nu_{t}=30 \mathrm{GeV}$ and $400 \mathrm{GeV} \leq m_{\pi_{t}} \leq 800 \mathrm{GeV}$, there will be $3.6 \times 10^{4} \sim 1.3 \times 10^{6}$ 
$\pi_{t}^{0}$ events to be generated per year at the $L H C$ with $\sqrt{s}=14 T e V$ and $\mathcal{L}_{\text {int }}=100 \mathrm{fb}^{-1}$.

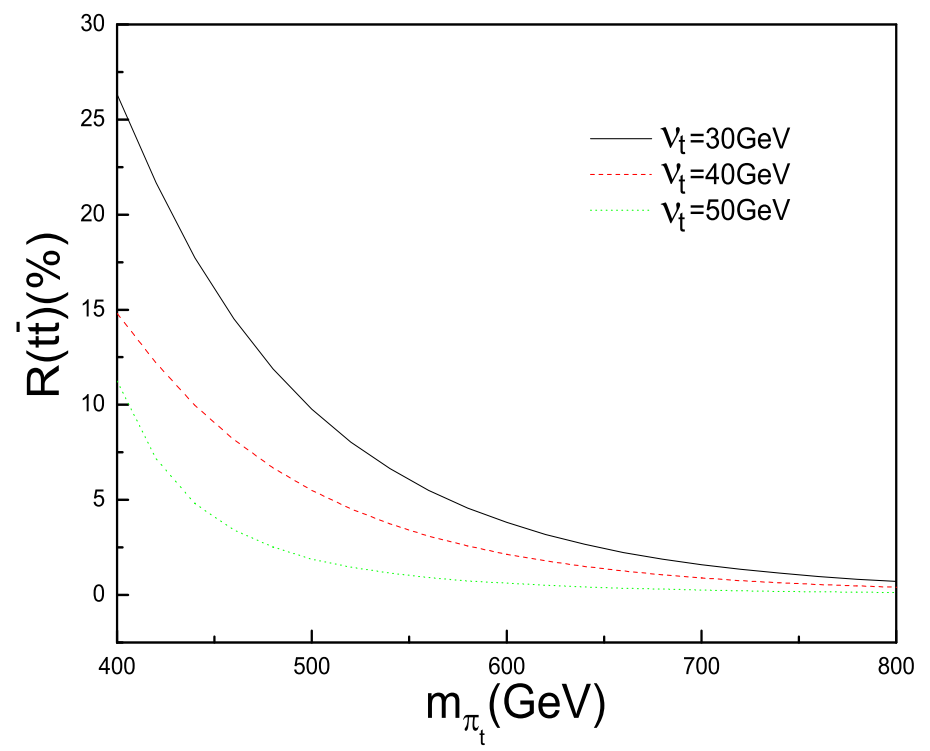

Figure 3: The relative correction parameter $R(t \bar{t})$ versus the mass parameter $m_{\pi_{t}}$ for $\nu_{t}=30 \mathrm{GeV}($ solid line $), \nu_{t}=40 \mathrm{GeV}($ dashed line $), \nu_{t}=50 \mathrm{GeV}($ dotted line $)$.

The production cross section of the top quark pair $(t \bar{t})$ has been calculated at nextto-next-to-leading(NNL) order in the context of the SM and can be measured to several percent accuracy at the $\mathrm{LHC}[13,14]$. Thus, it is needed to calculate the corrections of new physics beyond the SM to the $t \bar{t}$ production cross section. The neutral top-pion $\pi_{t}^{0}$ has large coupling with the top quarks and should generate significant corrections to the $t \bar{t}$ production cross section at the LHC via the enhanced gluon and bottom quark fusion mechanisms. The relative correction parameter $R(t \bar{t})=\delta \sigma(t \bar{t}) / \sigma^{S M}(t \bar{t})$ with $\delta \sigma(t \bar{t})=$ $\sigma^{H T H}(t \bar{t})-\sigma^{S M}(t \bar{t})$, which comes from $\pi_{t}^{0}$ exchange, is plotted as a function of the toppion mass $m_{\pi_{t}}$ for three values of the $V E V \nu_{t}$ in Fig.3. One can see from Fig.3 that the relative correction parameter $R(t \bar{t})$ is sensitive to the mass parameter $m_{\pi_{t}}$ and its value quickly decreases as $m_{\pi_{t}}$ increasing. However, as long as $m_{\pi_{t}} \leq 500 \mathrm{GeV}$ and $\nu_{t} / \nu \leq 1 / 5$, the value of $R(t \bar{t})$ is larger than $1.9 \%$, which might be detected at the LHC experiments.

To further discuss the possible signatures of the neutral top-pion $\pi_{t}^{0}$, we calculate the production cross section of the process $p p \rightarrow \pi_{t}^{0}+X \rightarrow \gamma \gamma+X$ at the LHC with 
$\sqrt{s}=14 T e V$. Our numerical results show that the production cross section $\sigma(\gamma \gamma)$ is very small. Even for $m_{\pi_{t}}=400 \mathrm{GeV}$ and $\nu_{t} / \nu \leq 1 / 8$, there will be only about $25 \gamma \gamma$ events to be generated per year at the LHC with $\sqrt{s}=14 T e V$ and $\mathcal{L}_{\text {int }}=100 \mathrm{fb}^{-1}$. This is because, compared to other decay modes, the branching ratio $\operatorname{Br}\left(\pi_{t}^{0} \rightarrow \gamma \gamma\right)$ is very small. Thus, the possible signals of $\pi_{t}^{0}$ predicted by the HTH model can not be detected via the process $p p \rightarrow \pi_{t}^{0}+X \rightarrow \gamma \gamma+X$ at the LHC experiments.

\section{Production of the charged top-pions $\pi_{t}^{ \pm}$at the LHC}

A charged scalar does not belong to the particle spectrum of the SM. The discovery of a charged scalar would be a clear signal for the existence of new physics beyond the SM. At the LHC, the main production mechanism of a light charged scalar is via the top quark pair production processes $g g(q \bar{q}) \rightarrow t \bar{t}$ followed by the top(or anti-top) quark decay, while, for a heavy charged scalar, the main production process at the leading order comes from the gluon bottom quark fusion process[15]. The complete NLO QCD corrections have been calculated by Ref.[16]. In the context of the topcolor-assisted technicolor(TC2) models[17], we have studied the production of the charged top-pions $\pi_{t}^{ \pm}$via the parton process $g b \rightarrow t \pi_{t}^{ \pm}$and discussed the possibility of detecting $\pi_{t}^{ \pm}$at the LHC via the $t b(\bar{t} b)$ decay channels[18]. The charged top-pions $\pi_{t}^{ \pm}$predicted by the HTH model have similar features to those from the TC2 models. In the framework of the HTH model, the production cross section $\sigma\left(t \pi_{t}^{-}\right)$for the process $p p \rightarrow g b+X \rightarrow t \pi_{t}^{-}+X$ at the LHC are plotted as a function of $m_{\pi_{t}}$ for three values of the top-Higgs $V E V \nu_{t}$ in Fig.4. For $400 \mathrm{GeV} \leq m_{\pi_{t}} \leq 800 \mathrm{GeV}$ and $\nu_{t}=50 \mathrm{GeV}$, the value of the cross section $\sigma\left(t \pi_{t}^{-}\right)$is in the range of $1755 \mathrm{fb} \sim 63 \mathrm{fb}$.

From the discussions given in section II, we can see that, for the charged top-pion $\pi_{t}^{-}$, the main decay mode is $\bar{t} b$. Thus, the associated production of the charged top-pion $\pi_{t}^{-}$with single top quark can easily transfer to the $t \bar{t} b$ final state. Our numerical results show that, for $m_{\pi_{t}}=600 \mathrm{GeV}$ and $\nu_{t} / \nu \approx 1 / 5$, there will be $2.8 \times 10^{4} t \bar{t} b$ events to be generated per year at the $\mathrm{LHC}$ with $\mathcal{L}_{\text {int }}=100 \mathrm{fb}^{-1}$. According the analysis conclusions for the backgrounds of the process $p p \rightarrow t \bar{t} b+X$ given by Ref.[15,19], we expect that the charged top-pions $\pi_{t}^{ \pm}$should be observed via the process $p p \rightarrow t \pi_{t}^{ \pm}+X \rightarrow t \bar{t} b(\bar{t} t \bar{b})+X$ 
in the near future LHC experiments.

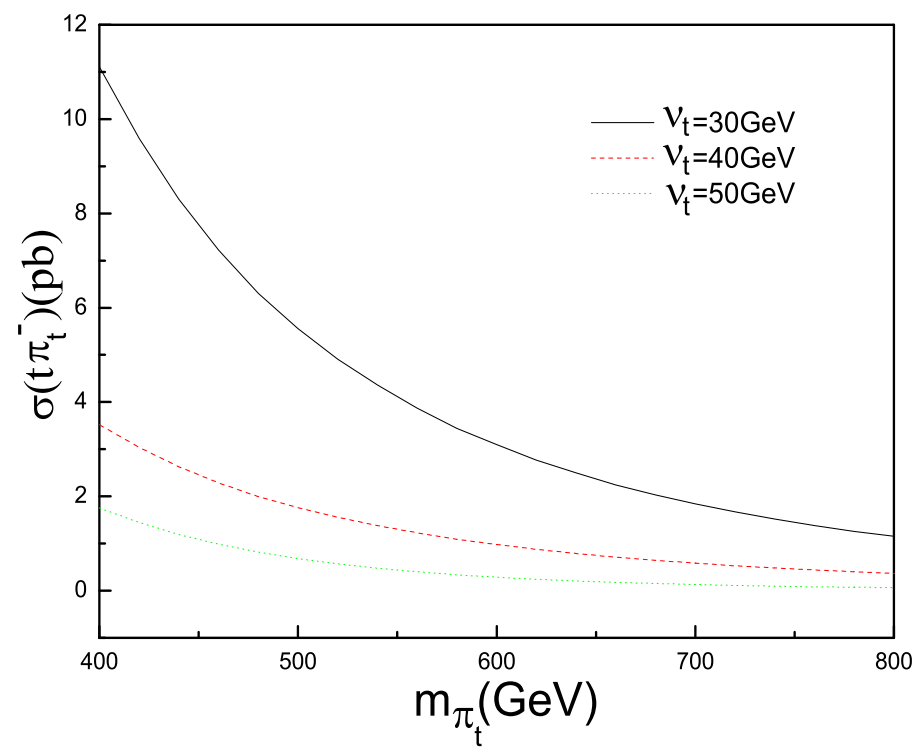

Figure 4: The production cross section $\sigma\left(t \pi_{t}^{-}\right)$as a function of the top-pion mass $m_{\pi_{t}}$ for $\nu_{t}=30 \mathrm{GeV}$ (solid line), $\nu_{t}=40 \mathrm{GeV}$ (dashed line), $\nu_{t}=50 \mathrm{GeV}$ (dotted line).

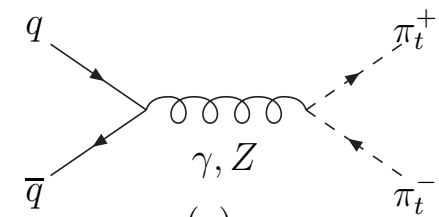

(a)

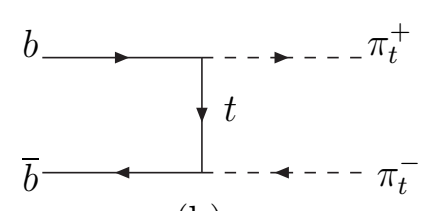

(b)

Figure 5: The leading order Feynman diagrams for $q \bar{q} \rightarrow \pi_{t}^{+} \pi_{t}^{-}$

At the LHC, the charged top-pions $\pi_{t}^{ \pm}$can also be produced in pair production mode. The main production processes are the usual Drell-Yan processes $q \bar{q} \rightarrow \pi_{t}^{+} \pi_{t}^{-}(q=u, d, c$, $s$ and $b$ ) through the s-channel $\mathrm{Z}$ exchange and photon exchange, and the t-channel bottom quark scatting process $b \bar{b} \rightarrow \pi_{t}^{+} \pi_{t}^{-}$, as shown in Fig.5. Certainly, $\pi_{t}^{+} \pi_{t}^{-}$production receives an additional contribution from the $g g$ fusion process $g g \rightarrow \pi_{t}^{+} \pi_{t}^{-}$at one loop. However, its contributions are very smaller than those of the tree level processes. Thus, we will ignore the process $g g \rightarrow \pi_{t}^{+} \pi_{t}^{-}$in the following estimation.

Using Eq.(3) and other relevant Feynman rules, we can write the invariant amplitude 
for the parton process $q\left(P_{1}\right)+\bar{q}\left(P_{2}\right) \rightarrow \pi_{t}^{+}\left(P_{3}\right)+\pi_{t}^{-}\left(P_{4}\right)$ as:

$$
M=M_{s}+M_{t}
$$

For the process $b\left(P_{1}\right)+\bar{b}\left(P_{2}\right) \rightarrow \pi_{t}^{+}\left(P_{3}\right)+\pi_{t}^{-}\left(P_{4}\right)$, the invariant amplitude comes from Fig.5(a) and (b):

$$
\begin{aligned}
M= & e \bar{v}\left(P_{2}\right) Q \gamma_{\nu} u\left(P_{1}\right) g^{\mu \nu}\left(P_{4}-P_{3}\right)_{\mu} \\
& +\bar{v}\left(P_{2}\right) \gamma_{\nu}\left(P_{L} g_{L}+P_{R} g_{R}\right) u\left(P_{1}\right) \frac{g^{\mu \nu}}{\hat{s}-m_{Z}^{2}} \frac{e}{S_{W} C_{W}}\left(P_{4}-P_{3}\right)_{\mu} \\
& +\frac{e^{2}}{2 m_{W}^{2} S_{W}^{2}}\left(\frac{v}{v_{t}}\right)^{2} \bar{v}\left(P_{2}\right)\left(m_{t} P_{R}+m_{b} P_{L}\right) \frac{\not q+m_{t}}{\hat{t}-m_{t}^{2}}\left(m_{t} P_{R}+m_{b} P_{L}\right) u\left(P_{1}\right) .
\end{aligned}
$$

For $u, c, d$ and $s$ quarks, we only consider the contributions of the s-channel process to the scattering amplitude, which can be written as:

$$
\begin{aligned}
M= & e \bar{v}\left(P_{2}\right) Q \gamma_{\nu} u\left(P_{1}\right) g^{\mu \nu}\left(P_{4}-P_{3}\right)_{\mu} \\
& +\bar{v}\left(P_{2}\right) \gamma_{\nu}\left(P_{L} g_{L}+P_{R} g_{R}\right) u\left(P_{1}\right) \frac{g^{\mu \nu}}{\hat{s}-m_{Z}^{2}} \frac{e}{S_{W} C_{W}}\left(P_{4}-P_{3}\right)_{\mu}
\end{aligned}
$$

with

$$
\begin{gathered}
Q=\frac{2}{3} e, \quad g_{L}=\frac{e}{4 S_{W} C_{W}}\left(2-\frac{8}{3} S_{W}^{2}\right), \quad g_{R}=-\frac{2 e S_{W}}{3 C_{W}}(q=u, c), \\
Q=-\frac{1}{3} e, \quad g_{L}=\frac{e}{4 S_{W} C_{W}}\left(-2+\frac{4}{3} S_{W}^{2}\right), \quad g_{R}=\frac{e S_{W}}{3 C_{W}}(q=d, s, b) .
\end{gathered}
$$

Where $\hat{s}=\left(P_{1}+P_{2}\right)^{2}$ and $\hat{t}=\left(P_{1}-P_{3}\right)^{2}$ are the usual Mandelstam variables. We have neglected the light quark masses in our calculation, except for the bottom quark mass in the couplings $\pi_{t}^{ \pm} t b$. Our numerical results are shown in Fig.6, in which we plot the production cross section $\sigma\left(\pi_{t}^{+} \pi_{t}^{-}\right)$for the process $p p \rightarrow \pi_{t}^{+} \pi_{t}^{-}+X$ at the LHC with $\sqrt{s}=14 T e V$ as a function of the top-pion mass $m_{\pi_{t}}$ for $\nu_{t} / \nu \approx 1 / 5$. To comparison, we use the solid line and dashed line to represent the contributions of the process $q \bar{q} \rightarrow$ $\pi_{t}^{+} \pi_{t}^{-}(q=u, c, d$ and $s)$ and the process $b b \rightarrow \pi_{t}^{+} \pi_{t}^{-}$, respectively. From Fig.6 one can see that the production cross section of the charged top-pion pair $\pi_{t}^{+} \pi_{t}^{-}$mainly comes from the usual Drell-Yan process $q \bar{q} \rightarrow \pi_{t}^{+} \pi_{t}^{-}(q=u, c, d$ and $s)$ through the s-channel 
$\mathrm{Z}$ exchange and photon exchange. The total production cross section $\sigma\left(\pi_{t}^{+} \pi_{t}^{-}\right)$is in the range of $12.5 \mathrm{fb} \sim 4.4 \mathrm{fb}$ for $400 \mathrm{GeV} \leq m_{\pi_{t}} \leq 800 \mathrm{GeV}$ and $\nu_{t} / \nu \approx 1 / 5$. The charged Higgs boson pair production at the LHC has been calculated to next-to-leading order[20]. They have shown that the total cross section for the process $p p \rightarrow H^{+} H^{-}+X$ is smaller than $10 \mathrm{fb}$ for $\tan \beta \leq 30$ and $m_{H} \geq 400 \mathrm{GeV}$. Thus, we expect that the charged top-pions $\pi_{t}^{ \pm}$predicted by the HTH model can be more easy detected at the LHC via this process than that for the charged Higgs bosons $H^{ \pm}$.

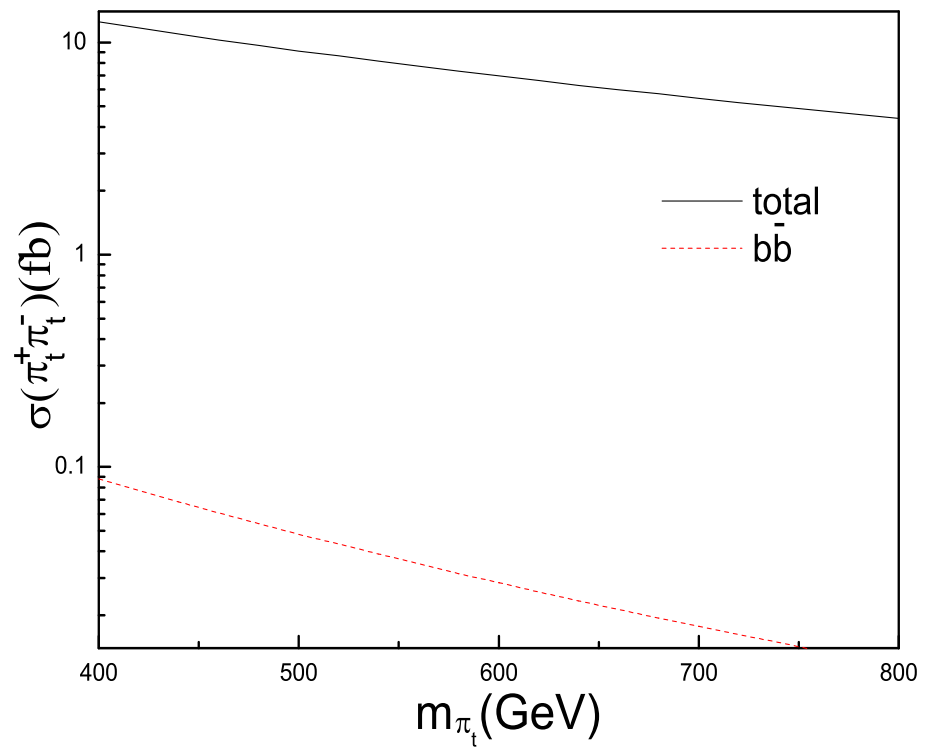

Figure 6: The cross section $\sigma\left(\pi_{t}^{+} \pi_{t}^{-}\right)$as a function of the top-pion mass $m_{\pi_{t}}$ for $\nu_{t} / \nu \approx 1 / 5$.

\section{Conclusions and discussions}

Since extra dimensions can play an active role in the physics of the TeV scales, theories with extra dimensions have recently attracted enormous attention[21]. Based on $S U(2) \times S U(2) \times U(1)$ gauge theory in slices of two "back-to-back" $A d S_{5}^{\prime} s$, topcolor theories were reconsidered in extra dimensions[5]. For extra dimensional descriptions of topcolor theories, there are three possibilities for EWSB: Higgs-top-Higgs, higgsless-topHiggs, and higgsless-higgsless. The higgsless-top-Higgs(HTH) model which is similar to TC2 models, predicts the existence of the top-pions $\pi_{t}^{0, \pm}$ and the top-Higgs boson $h_{t}^{0}$. Since the top-Higgs boson $h_{t}^{0}$ is mainly responsible for generating the large top mass and 
gives small contributions to EWSB, these new particles $\left(\pi_{t}^{0, \pm}, h_{t}^{0}\right)$ have large Yukawa couplings to the third generation quarks, which might produce characteristic signatures at various high-energy collider experiments.

In this paper, we discuss the production of the top-pions $\pi_{t}^{0, \pm}$ predicted by the HTH model at the LHC with $\sqrt{s}=14 T e V$ and $\mathcal{L}_{\text {int }}=100 \mathrm{fb}^{-1}$ via various suitable mechanisms (gluon-gluon fusion, bottom-bottom fusion, gluon-bottom fusion, and the usual Drell-Yan processes). The following conclusions are obtained.

(1) The neutral top-pion $\pi_{t}^{0}$ can be abundantly produced via the gluon-gluon fusion and bottom-bottom fusion processes. The production cross section $\sigma\left(\pi_{t}^{0}\right)$ is sensitive to the top-pion mass $m_{\pi_{t}}$ and the top-Higgs $V E V \nu_{t}$, which mainly comes from the parton process $g g \rightarrow \pi_{t}^{0}$. For $\nu_{t} \approx 50 \mathrm{GeV}$ and $400 \mathrm{GeV} \leq m_{\pi_{t}} \leq 800 \mathrm{GeV}$, the value of $\sigma\left(\pi_{t}^{0}\right)$ is in the range $130 \mathrm{fb} \sim 4800 \mathrm{fb}$.

(2) The main decay modes of the heavy neutral top-pion $\pi_{t}^{0}$ are $t \bar{t}$ and $g g$, thus the neutral top-pion $\pi_{t}^{0}$ can generate significant corrections to the $t \bar{t}$ production at the LHC via the process $p p \rightarrow \pi_{t}^{0}+X \rightarrow t \bar{t}+X$. For $\nu_{t}=40 \mathrm{GeV}$ and $400 \mathrm{GeV} \leq m_{\pi_{t}} \leq 600 \mathrm{GeV}$, the value of the relative correction parameter $R(t \bar{t})$ is in the range of $2.1 \% \sim 14.8 \%$.

(3) For a heavy charged scalar, the dominant production process at the LHC is its associated production with a top quark via gluon bottom fusion. The LHC has good potential for discovering a heavy charged scalar through this process. Thus, we estimate the production cross section of the process $p p \rightarrow t \pi_{t}^{-}+X$ at the LHC with $\sqrt{s}=14 T e V$ and $\mathcal{L}_{\text {int }}=100 \mathrm{fb}^{-1}$. Our numerical results show that the production rate is significantly large and the possible signals of $\pi_{t}^{ \pm}$might be detected at the LHC through the process $p p \rightarrow t \pi_{t}^{ \pm}+X$ in their $t \bar{b}(\bar{t} b)$ decay modes.

(4) As long as the charged scalar is not too heavy, it is possible to be produced in pair at the near future LHC experiments. The main production processes are the usual DrellYan processes and the $t$-channel bottom quark scattering process. We further estimate the rate of the charged top-pion pair production at the LHC. Our numerical results show that the production cross section $\sigma\left(\pi_{t}^{+} \pi_{t}^{-}\right)$is in the range of $9.1 \mathrm{fb} \sim 18.6 \mathrm{fb}$ for $\nu_{t}=30 \mathrm{GeV}$ and $400 \mathrm{GeV} \leq m_{\pi_{t}} \leq 800 \mathrm{GeV}$, which might be larger than that for the charged Higgs bosons 
$H^{ \pm}\left(m_{H} \geq 400 \mathrm{GeV}\right.$ and $\left.\tan \beta \leq 30\right)$ predicted the minimal supersymmetric standard model.

In conclusion, as long as the top-pions $\pi_{t}^{0, \pm}$ are not too heavy $\left(m_{\pi_{t}}<1 T e V\right)$, they can be abundantly produced at the LHC via various suitable mechanisms. The possible signatures of these new particles might be detected at the LHC experiments.

At last, we have to say that all of our numerical results are obtained at leading order. The strong Yukawa couplings can give large corrections to these numerical results. For example, for the process $p p \rightarrow g b+X \rightarrow t \pi_{t}^{-}+X$, the leading order cross section given in section IV can be enhanced about $80 \%$ by the effects of next leading corrections. Thus, the numerical results given in this paper can only be taken as order-of-magnitude guides. However, we expect that our estimations can provide the right qualitative picture.

\section{Acknowledgments}

This work was supported in part by Program for New Century Excellent Talents in University(NCET-04-0290), the National Natural Science Foundation of China under the Grants No.10475037. 


\section{References}

[1] C. T. Hill and E. H. Simmons, Phys. Rept. 381(2003)235; [Erratum-ibid, $390(2004) 553]$.

[2] N. Arkani-Hamed, S. Dimopoulos and G. R. Dvali, Phys. Lett. B429(1998)263; I. Antoniadis, N. Arkani-Hamed, S. Dimopoulos and G. R. Dvali, Phys. Lett. B436(1998)257; K. R. Dienes, E. Dudas and T.Gherghetta, Phys. Lett. B436(1998)55; Nucl. Phys. B537(1999)47.

[3] Y. Kavamura, Prog. Theor. Phys. 103(2000)613; Prog. Theor. Phys. 105(2001)691; A. Hebecker and J. March-Russell, Nucl. Phys. B613(2001)3; Nucl. Phys. B625(2002)128; L. J. Hall, Y. Nomura, T. Okui and D. R. Smith, Phys. Rev. D65(2002)035008; L. J. Hall and Y. Nomura, Phys. Rev. D65(2002)125012.

[4] C. Csaki, C. Grojean, L. Pilo and J. Terning, Phys. Rev. Lett. 92(2004)101802; Y. Nomura, JHEP 0311(2003)050; R. Barbieri, A. Pomarol and R. Rattazzi, Phys. Lett. B591(2004)141; C. Csaki, C. Grojean, J. Hubisz, Y. Shirman and J. Terning, Phys. Rev. D70(2004)015012.

[5] G. Cacciapaglia et al., Phys. Rev. D72(2005)095018.

[6] N. Arkani-Hamed, H. C. Cheng, B. A. Dobrescu and L. J. Hall, Phys. Rev. D 62(2000)096006; M. Hashimoto and D. K. Hong, Phys. Rev. D71(2005)056004.

[7] G. Passarino and M. Veltman, Nucl. Phys. B160(1997)151; A. Axelrod, Nucl. Phys. B209(1982)349; M. Clements et al., Phys. Rev. D44(1983)570.

[8] The CDF Collaboration, the D0 Collaboration, the Tevatron Electroweak Working Group, Combination of CDF and DO Result on the Top-Quark mass, hep-ex/0507091.

[9] S. Eiddman et al. [Particle data Group], Phys. Lett. B592 (2004)1.

[10] X. L. Wang, W. N. Xu, L. L. Du, Commun. Theor. Phys. 41(2004)737. 
[11] J. Pumplin et al. JHEP 0207(2002)012; D. Stump et al., JHEP 0310(2003)046.

[12] T. Plehn, Phys. Rev. D67(2003)014018; F. Maltoni, Z. Sullivan and S. Willenbrock, Phys. Rev. D67(2003)093005; R. V. Harlander and W. B. Kilgore, Phys. Rev. D68(2003)013001; A. Alves and T. Plehn, Phys. Rev. D71(2005)115014.

[13] ATLAS Collaboration, Technical Design Report, CERN-LHCC-99-15; CMS Collaboration, Technical Proposal, CERN-LHCC-94-38; G. Weiglein et al. [LHC/LC Study Group], hep-ph/0410364.

[14] M. Beneke et al., "Top Quark Physics", hep-ph/0003033, D. Chakrahorty, J. Konigsherg and D. Rainwater, Ann. Rev. Nucl. Part. Sci. 53(2003)301; W. Wagner. Rept. Prog. Phys. 68(2005)2409.

[15] A. C. Bawa, C. S. Kim and A. D. Martin, Z. Phys. C47(1990)75; J. F. Gunion, Phys. Lett. B322(1994)125; V. D. Barger, R. J. N. Phillips and D. P. Roy, Phys. Lett. B324(1994)236.

[16] S. H. Zhu, Phys. Rev. D67(2003)075006; T. Plehn, Phys. Rev. D67(2003)014018; E. L. Berger, T. Han, J. Jiang, T. Plehn, Phys. Rev. D71(2005)115012.

[17] C. T. Hill, Phys. Lett. B345(1995)483; K. D. Lane and E. Eichten, Phys. Lett. B 352(1995)382; K. D. Lane, Phys. Lett. B433(1998)96; G. Cvetic, Rev. Mod. Phys. 71(1999)513.

[18] Chong-Xing Yue, Zheng-Jun Zong, Li-Li Xu and Jian-Xing Chen, Phys. Rev. D73(2006)015006.

[19] N. Kidonakis, R. Vogt, Int. J. Mod. Phys.A20(2005)3171; D. P. Roy, hep-ph/0510070.

[20] J. F. Gunion et al., Nucl. Phys. B294(1987)621; A. Krause, T. Plehn, M. Spira and P. M. Zerwas, Nucl. Phys. B519(1998)85; A. A. Barrientos Bendezu and B. A. Kniehl, Nucl. Phys. B568(2000)305; S. Moretti and J. Rathsman, Nucl. Phys. J. 
C33(2004)41; H. S. Hou et al., Phys. Rev. D71(2005)075014; A. Alves and T. Plehn, Phys. Rev. D71(2005)115014.

[21] C. Csaki, hep-ph/0404096; C. Csaki, J. Hubisz and P. Meade, hep-ph/0510275. 